\title{
Placebo controlled trial of enteric coated pancreatin microsphere treatment in patients with unresectable cancer of the pancreatic head region
}

\author{
M J Bruno, E B Haverkort, G P Tijssen, G N J Tytgat, D J van Leeuwen
}

\begin{abstract}
Background-Impeded flow of pancreatic juice due to mechanical obstruction of the pancreatic duct in patients with cancer of the pancreatic head region causes exocrine pancreatic insufficiency with steatorrhoea and creatorrhoea. This may contribute to the profound weight loss that often occurs in these patients.

Aims-To investigate whether pancreatic enzyme replacement therapy prevents this weight loss.

Patients-Twenty one patients with unresectable cancer of the pancreatic head region with suspected pancreatic duct obstruction, a biliary endoprosthesis in situ, and a Karnofsky performance status greater than 60 .

Methods-Randomised double blind trial of eight weeks with either placebo or high dose enteric coated pancreatin enzyme supplementation. All patients received dietary counselling.

Results-The mean difference in the percentage change of body weight was $4.9 \%$ $(p=0.02,95 \%$ confidence interval for the difference: 0.9 to 8.9$)$. Patients on pancreatic enzymes gained $1.2 \%(0.7 \mathrm{~kg})$ body weight whereas patients on placebo lost $3.7 \%(2.2 \mathrm{~kg})$. The fat absorption coefficient in patients on pancreatic enzymes improved by $12 \%$ whereas in placebo patients it dropped by $8 \%(p=0.13,95 \%$ confidence interval for the difference: -6 to 45$)$. The daily total energy intake was 8.42 $\mathrm{MJ}$ in patients on pancreatic enzymes and 6.66 MJ in placebo patients $(p=0.04$, $95 \%$ confidence interval for the difference: 0.08 to 3.44).

Conclusions-Weight loss in patients with unresectable cancer of the pancreatic head region and occlusion of the pancreatic duct can be prevented, at least for the period immediately after insertion of a biliary endoprosthesis, by high dose enteric coated pancreatin enzyme supplementation in combination with dietary counselling.

(Gut 1998;42:92-96)
\end{abstract}

Keywords: pancreatic cancer; weight loss; pancreatic enzyme therapy; enteric coated enzyme therapy; palliation; dietary counselling

Cancer of the pancreatic head region is associated with an extremely poor prognosis. Carcinomas of pancreatic origin, which represent about $85 \%$ of these cancers, have the worst overall prognosis with fewer than $3 \%$ of affected patients alive five years after the initial diagnosis. ${ }^{12}$ It is the fourth leading cause of death from cancer in the United States. The median survival for locally unresectable disease is 33 weeks and for advanced metastatic disease is 10 weeks. $^{3}$ Only $10-20 \%$ of all patients are eligible for potentially curative resection. Consequently, $80-90 \%$ of patients have locally unresectable or advanced metastatic disease and for these patients only palliative treatment options remain. Symptoms include obstructive jaundice, duodenal obstruction, pain, and weight loss. Palliative treatment is mainly directed against the former three. Interventions to prevent (further) weight loss have as yet received little or no attention, in spite of the frequent occurrence of this symptom. About $90 \%$ of patients with pancreatic carcinoma have weight loss at the time of diagnosis. ${ }^{4}$ Weight loss in cancer is caused by primary and secondary tumour effects. ${ }^{56}$ Primary tumour effects causing metabolic abnormalities include increased glucose production, increased whole body protein breakdown, and increased lipolysis and depletion of body fat stores. $^{7}$ These effects can be augmented by secondary tumour effects such as intestinal obstruction due to tumour expansion, or side effects of treatment such as nausea induced by chemotherapy. Another example of such a secondary effect is impeded flow of pancreatic juice due to mechanical obstruction of the pancreatic duct in patients with cancer of the pancreatic head region. This may cause exocrine pancreatic insufficiency with faecal losses of energy through steatorrhoea and creatorrhoea. Such secondary effects should potentially be reversible if the underlying pathophysiological mechanism is understood and if treatment options are available. Weight loss in pancreatic cancer is associated with maldigestion due to pancreatic duct obstruction and pancreatic enzyme replacement therapy results in a significant improvement in fat and protein absorption. ${ }^{89}$ However, studies evaluating the long term efficacy of pancreatic enzyme replacement therapy are lacking to date. We conducted a prospective, randomised, placebo controlled trial to test the hypothesis that weight loss in patients with unresectable cancer of the pancreatic head region with occlusion of the pancreatic duct can be reduced or prevented by pancreatic enzyme replacement therapy in combination with dietary counselling. 
Methods

Patients were enrolled in this single centre, double blind, placebo controlled, randomised trial from January 1993 to August 1994. All patients gave written informed consent. The trial was approved by the Medical Ethics Committee of the Academic Medical Centre.

\section{PATIENT SELECTION}

Consecutive patients in whom a biliary endoprosthesis was inserted because of obstructive jaundice due to cancer of the pancreatic head region were screened for inclusion into the trial. Criteria for trial entry were: clinical presentation (medical history, physical examination, and information from imaging studies) compatible with cancer of the pancreatic head region (pancreatic, distal common bile duct, ampullary, or duodenal carcinoma) preferably proven by cytology or histology; obstruction of the common bile duct proven by endoscopic retrograde cholangiopancreaticography; obstruction of the pancreatic duct with less than 2 $\mathrm{cm}$ filling of the distal duct, or no filling despite multiple attempts; not eligible for resectional surgery because of poor general condition, local unresectability, or advanced disease with metastases; a patent biliary endoprosthesis at trial entry as assessed by medical history (pruritus, colour of urine, and colour of faeces), physical examination (jaundice) and blood samples (values of plasma bilirubin, plasma alkaline phosphatase, and plasma $\gamma$-glutamyltransferase); and a Karnofsky performance status greater than 60 .

Exclusion criteria were: history of major gastrointestinal surgery; history of chronic gastrointestinal disease (for example, coeliac disease, Crohn's disease); coexistent other primary malignancy; radiotherapeutic or cytostatic treatment; and any use of antacids, mucosal protective agents, $\mathrm{H}_{2}$ receptor antagonists, or proton pump inhibitors that could not be discontinued.

With a minimum of two weeks after endoscopic retrograde cholangiopancreaticography, eligible patients who gave informed consent visited the outpatient clinic or were visited at home by a physician and a dietitian. Both the physician and the dietitian were the same individuals for all patients throughout the trial. During this entry visit, blood samples were taken to assess the patency of the biliary endoprosthesis. The week following this entry visit, the fat absorption coefficient without trial medication was assessed. For this, patients recorded their daily dietary intake during six consecutive days in preprinted diaries of dietary intake and collected stools during the last three days of this period. For calculation of the fat absorption coefficient, the mean daily fat intake and the main daily faecal fat excretion were used.

TREATMENT AND RANDOMISATION

Randomisation took place one week after the entry visit. Patients were randomised to receive either pancreatic enzyme replacement therapy or placebo. The pancreatic enzyme preparation under investigation was Panzytrat 25000
(Nordmark GmbH, Uetersen, Germany), an enteric coated pancreatin microsphere preparation containing 25000 PhEur units of lipase, 1250 PhEur units of proteases, and 22500 PhEur units of amylase per capsule. The placebo matched the active drug in appearance, taste, and weight and contained pharmacologically inactive substances. Patients used two capsules during main meals and one capsule during in between snacks. Capsules were swallowed whole.

After randomisation, a double blind trial period of eight weeks started. If a patient lost more than $5 \mathrm{~kg}$ after the first four weeks of treatment, unblinding took place. This was defined by the Medical Ethical Committee of our hospital as the upper limit for continuation of the study while having the chance that the patient had this weight loss due to exocrine pancreatic insufficiency while receiving placebo. If a patient failed to complete the double blind trial period of eight weeks, the outcome obtained after four weeks of treatment was used in the analysis without further adjustment. This was the case in one patient only.

After the double blind trial period, patients were followed for an additional four weeks in an open descriptive trial period. Patients on placebo were switched to pancreatic enzymes two capsules three times daily during main meals and one capsule three times daily during in between snacks. In patients who were already on pancreatic enzymes during the double blind trial period, the dose was lowered to one capsule three times daily during the main meals.

Throughout the trial, all patients received dietary counselling from an experienced dietitian. During the visits and weekly telephone contacts it was attempted to optimise the intake of calories and nutrients. Patients were encouraged to take as much fat as they could tolerate. Patients were also encouraged to divide the energy intake over six meals daily: three main meals and three in between snacks. No strict rules or prohibitions with respect to dietary intake were imposed.

\section{FOLLOW UP}

Follow up visits were scheduled at four, eight, and 12 weeks after the randomisation visit. During each visit, body weight was measured. The occurrence and severity of complaints and symptoms associated with steatorrhoea were assessed by means of the questionnaire. This questionnaire had free field and multiple point ratings that included scores for frequency of stools (free field), consistency of stools (three point rating: moulded/pappy/diarrhoea), amount of stools compared with preillness period (five point rating from much less to much more), occurrence of foul smelling stools (six point rating ranging from never to always), whether stools stuck in the toilet (six point rating ranging from never to always), occurrence of abdominal cramps (five point rating ranging from never to very often), and the occurrence of flatulence (five point rating ranging from never to very often). Patients were also questioned about potential side effects. Prior to 
Table 1 Baseline patient characteristics

\begin{tabular}{lll}
\hline Baseline patient characteristics & Placebo & Pancreatic enzyme therapy \\
\hline Number of patients & 10 & 11 \\
Men/women & $4 / 6$ & $4 / 7$ \\
Age (y) & $79(9)$ & $73(11)$ \\
Weight loss (kg) & $-12.9(9.1)$ & $-9.9(5.7)$ \\
Body weight (kg) & $58.1(8.1)$ & $59.0(5.2)$ \\
Type of cancer (clinical diagnosis) & & \\
$\quad$ Pancreatic carcinoma & 9 & 10 \\
$\quad$ Distal common bile duct carcinoma & 1 & 0 \\
Ampullary carcinoma & 0 & 1 \\
Tumour size (cm) $)^{\star}$ & $3.9(1.0)$ & $3.5(1.3)$ \\
Presence of liver metastases & 1 & 0 \\
Presence of lung metastases & 0 & 0 \\
Fat absorption coefficient & $72(24)$ & $70(28)$ \\
Karnofsky performance status & $78(10)$ & $80(11)$ \\
Diabetes mellitus & 3 & 2 \\
\hline
\end{tabular}

^Mean (SD).

For all items $p>0.05$. priate $t$ tests or non-parametric tests were used. When appropriate, the $95 \%$ confidence interval for the difference was calculated. For categorical data, the $\chi^{2}$ test for trend was used. Differences between the groups were considered significant if the $\mathrm{p}$ value was less than 0.05 for a two tailed test.

\section{Results}

TRIAL POPULATION

Twenty four patients entered the trial. Two patients died before data regarding the effect of treatment could be obtained. One patient discontinued participation after two weeks following a hospital admission for a stent exchange. Twenty one patients were available for analysis of the double blind treatment period. The groups were comparable at the time of randomisation (for all items $\mathrm{p}>0.05$ ) (table 1).

During the double blind trial period one patient (on pancreatic enzymes) developed diabetes and two patients required exchange of an occluded biliary stent (one patient on placebo and one on pancreatic enzymes). At the time of the preparation of the manuscript, all patients had died.

Medication compliance during the double blind trial period was comparable between both groups: patients on placebo had a median intake of 7.8 capsules per day (range: 4.6-9.0) and patients on the pancreatic enzyme preparation a median intake of 8.0 capsules per day (range: 5.9-8.9) dietary intake of total calories, fat, protein, and carbohydrates during the double blind trial period; change in occurrence and severity of subjective symptoms and complaints associated with steatorrhoea during the double blind trial period; and occurrence and nature of adverse events.

ANALYSIS

The entry of patients was scheduled from January 1993 to August 1994. As the natural variation of weight change in patients with unresectable cancer of the pancreatic head region is unknown, formal power calculations could not be performed. An independent interim analysis was performed five months prior to the scheduled termination date of the trial. Based on this interim analysis, it was decided to terminate the trial at the scheduled date irrespective of the final outcomes.

All data were analysed according to the intention to treat principle. Quantitative data are expressed as means (SD) or as medians with the lower and upper quartiles given. For the comparison of continuous variables, appro-
DOUBLE BLIND TRIAL PERIOD

Table 2 lists treatment outcomes. The difference in the percentage change in body weight between both groups was statistically significant with a mean difference of $4.9 \%(p=0.02$, $95 \%$ confidence interval for the difference ranging from 0.9 to 8.9). Patients on pancreatic enzymes gained $1.2(4.3) \%$ body weight whereas patients on placebo lost $3.7(4.4) \%$. This was reflected by the fact that patients on pancreatic enzymes gained 0.7 (2.5) $\mathrm{kg}$ whereas patients on placebo lost $2.2(2.7) \mathrm{kg}$.

The difference between the two groups with respect to the change in the fat absorption coefficient was $20 \%(p=0.13,95 \%$ confidence interval for the difference ranging from -6 to 45). Fat absorption in patients on pancreatic enzymes increased by $12(25) \%$ whereas in patients on placebo it dropped by 8 (25)\%. Patients on pancreatic enzymes reached a significantly higher total daily energy intake (8.42 (1.88) MJ) than patients on placebo

Table 2 Treatment outcomes of primary and secondary end points

\begin{tabular}{llllll}
\hline Treatment parameter & Placebo $^{*}$ & $\begin{array}{l}\text { Pancreatic enzyme } \\
\text { therapy }\end{array}$ & p Value & $\begin{array}{l}\text { Mean } \\
\text { difference }\end{array}$ & $\begin{array}{l}\text { 95\% CI for the } \\
\text { difference }\end{array}$ \\
\hline Change in body weight (\%) & $-3.7(4.4)$ & $+1.2(4.3)$ & 0.02 & 4.9 & 0.9 to 8.9 \\
Change in body weight (kg) & $-2.2(2.7)$ & $+0.7(2.5)$ & 0.02 & 2.8 & 0.4 to 5.2 \\
Change in fat absorption coefficient & $-8(25)$ & $+12(25)$ & 0.13 & 20 & -6 to 45 \\
Change in stool frequency per day & $+0.2(1.0)$ & $-1.0(1.9)$ & 0.07 & 1.2 & NA \\
Daily total caloric intake (MJ) & $6.66(1.78)$ & $8.42(1.88)$ & 0.04 & 1.76 & 0.08 to 3.44 \\
Daily fat intake (MJ) & $2.65(0.94)$ & $3.31(1.07)$ & 0.15 & 0.66 & -0.26 to 1.58 \\
Daily protein intake (MJ) & $0.92(0.24)$ & $1.27(0.30)$ & $<0.01$ & 0.36 & 0.11 to 0.61 \\
Daily carbohydrate intake (MJ) & $3.10(0.95)$ & $3.81(1.00)$ & 0.11 & 0.71 & 0.18 to 1.61 \\
\hline
\end{tabular}

${ }^{\star}$ Mean (SD). 
(6.66 (1.78) MJ) ( $\mathrm{p}=0.04)$. The mean difference was $1.76 \mathrm{MJ}$ with a $95 \%$ confidence interval for the difference ranging from 0.08 to 3.44. The intake of fat, proteins, and carbohydrates was higher in patients using pancreatic enzymes than in patients on placebo, although only in the case of protein intake was this statistically significant. No adverse events were observed that could be attributed to the use of the trial medication. The mean changes in the severity and occurrence of steatorrhoea associated complaints between both groups were not significantly different. The stool frequency, however, showed a tendency to drop in patients on pancreatic enzymes (-1 (1.9)) compared with patients on placebo $(+0.2(1.0))$. Although not a formal end point of the study we also compared the change in Karnofsky performance status and found no significant difference.

\section{OPEN DESCRIPTIVE TRIAL PERIOD}

Seven patients did not complete the open descriptive trial period: two patients died, three patients were admitted to the hospital because of serious deterioration of their condition, one patient discontinued the trial in the terminal phase of his illness, and in one patient the family indicated that further cooperation would be too strenuous. Fourteen patients were available for analysis, six in the placebo group and eight in the pancreatic enzyme preparation group.

In patients who were switched from placebo to pancreatic enzymes in a dose of two capsules three times daily during the main meals and one capsule three times daily during in between snacks, mean body weight remained stable during the four weeks of treatment $(+0.1$ (1.5) $\mathrm{kg}$ ). During this period the mean total daily energy intake did not change substantially $(+0.20(0.86) \mathrm{MJ})$, but the occurrence and severity of steatorrhoea associated complaints showed a tendency towards improvement. In those patients already on pancreatic enzymes during the double blind trial period who were switched to a dose of one capsule three times daily during the main meals, one patient did not adhere to the lower dose and increased the number of capsules on his own initiative. In the remaining seven patients, the mean body weight showed a slight reduction $(-0.8$ (2.0) $\mathrm{kg})$. The mean total daily energy intake did not change substantially (+0.05 (0.49) MJ) and the occurrence and severity of steatorrhoea associated complaints showed a tendency towards worsening.

\section{Discussion}

The results of this trial indicate that pancreatic enzyme replacement therapy can (partly) prevent weight loss in patients with unresectable cancer of the pancreatic head region and occlusion of the pancreatic duct, at least in the initial period after the diagnosis is made and a biliary endoprosthesis is inserted. The mean difference in the percentage change in body weight between patients on pancreatic enzymes and patients on placebo over a treatment period of eight weeks was $4.9 \%$, corresponding to a mean difference in body weight of $2.8 \mathrm{~kg}$. There are various mechanisms by which the results of this trial may be explained.

Improvement of fat absorption leads to better digestion and hence to a reduction in energy loss through steatorrhoea. This energy loss may be quite considerable because one gram of fat contains $0.04 \mathrm{MJ}$ and one gram of protein contains $0.02 \mathrm{MJ}$. Fat absorption did show a trend in favour of the enteric coated pancreatin enzyme preparation but the difference was not statistically significant. However, we may have overestimated the fat absorption coefficient because we did not use a fixed fat intake to assess steatorrhoea. Ethically, we considered it unjustified to force terminally ill patients to go on a six day diet of $80-100 \mathrm{~g}$ of fat on two different occasions. A practical solution was found by having patients record their dietary intake for six consecutive days while collecting stools during the last three days, after analysis of which the mean daily fat intake was calculated. This implies, however, that for some patients fat intake was as low as $40-50 \mathrm{~g}$ daily. Many patients are capable of absorbing up to $50 \mathrm{~g}$ of fat without measurable pancreatic enzyme activity because of non-pancreatic sources of lipas $\mathrm{e}^{10}$; this may lead to an overestimation of the fat absorption coefficient and to an underestimation of the efficacy of pancreatic enzyme replacement therapy. Moreover, if creatorrhoea was present, which may well be the case in these patients, it may also have been improved by enteric coated pancreatin enzyme therapy. This is important because cancer patients often have a negative nitrogen balance and additional losses due to maldigestion would further increase the breakdown of muscle and fat tissue. In one study it was shown that treatment with pancreatic enzymes significantly improved moderate to severe fat or protein malabsorption in patients with pancreatic cancer. $^{8}$

Steatorrhoea associated complaints such as anorexia, early satiety, and bloating may in part be caused by the effect of unabsorbed fat on gastrointestinal motility with delayed gastric emptying and deceleration of small intestinal transit. ${ }^{11}$ Patients on pancreatic enzymes reached a significantly higher daily total energy intake than patients on placebo whereas the occurrence and severity of steatorrhoea associated complaints did not differ (and therefore was not higher in the patients on pancreatic enzymes despite a higher energy intake). For reasons not investigated in this study, it may well be that for these patients control of body weight had a higher priority than lowering digestive complaints.

The results of the open descriptive trial period emphasise the importance of providing an adequate dose of pancreatic enzymes which also need to be given during in between snacks. One patient did not adhere to three capsules per day and increased the number of capsules on his own initiative. In those patients who were switched to the lower dose of pancreatic enzymes, body weight showed a slight reduction compared with the previous visit and steatorrhoea associated symptoms worsened. 
For many patients with cancer, weight loss is an extremely depressing symptom. It affects the subjective well being of patients and may have consequences in terms of therapeutic options and survival. In a large group of patients with various primary cancer sites in the terminal stages of their illness (among them pancreatic cancer), weight loss, besides other symptoms such as performance status and problems with eating or anorexia, had a predictive value with respect to survival, independent of the primary tumour site. ${ }^{12}$ In our group of patients no further anticancer therapy was given. If, however, radiotherapy and/or chemotherapy are to be used in an attempt to improve the outcome in terms of quality of life and survival, a catabolic state should be avoided. Pancreatic enzyme therapy, in combination with dietary counselling, could play a role in maintaining an anabolic state as long as possible. Weight loss prior to chemotherapy was found to have a prognostic effect on survival in a range of different tumour types. ${ }^{13}$ In the case of advanced pancreatic cancer, the effect in the latter study was not statistically significant.

In summary, the results of this trial indicate that in patients with unresectable cancer of the pancreatic head region with occlusion of the pancreatic duct, weight loss can be partly prevented by the administration of a enteric coated pancreatin microsphere preparation in combination with dietary counselling, at least for the period immediately after diagnosis and insertion of a biliary endoprosthesis. The price of pancreatic enzyme supplementation per patient per day (mean intake of eight capsules per day) was $f 8.00$ ( $£ 2.60)$. In routine patient management, in addition to the cost of medication, dietary intervention and support would result in additional expense. In conclu- sion, pancreatic enzyme replacement therapy seems to be an additional and valuable tool in the palliation of this condition. As endoscopic pancreatic duct drainage by the insertion of an endoprosthesis is becoming increasingly feasible, future studies may compare the efficacy of pancreatic enzyme replacement therapy versus pancreatic duct drainage as well as the psychological effects of such intervention.

This study was supported financially by Knoll BV, Amsterdam, The Netherlands. Part of this work was presented at the Annual Meeting of the American Gastroenterological Association 1995, San Diego, USA.

1 Michelassi F, Erroi F, Dawson PJ, et al. Experience with 647 consecutive tumors of the duodenum, ampulla, head of the consecutive tumors of the duodenum, ampulla, head of the
pancreas, and distal common bile duct. Ann Surg pancreas, and dist

2 Gudjonsson B. Cancer of the pancreas. 50 years of surgery. Cancer 1987;60:2284-303.

3 Kalser MH, Barkin J, MacIntyre JM. Pancreatic cancer. Assessment of prognosis by clinical presentation. Cancer 1985;56:397-402.

4 Gullick HD. Carcinoma of the pancreas. Medicine 1959;38: $47-85$.

5 Heber D, Byerly LO, Chlebowski RT. Metabolic abnormalities in the cancer patient. Cancer 1985;55:225-9.

6 Heber D, Byerley LO, Chi J, et al. Pathophysiology of malnutrition in the adult cancer patient. Cancer 1986;58:
mats malnutrition.

7 Heber D, Tchelonedyian NS. Pathophysiology of cancer: hormonal and metabolic abnormalities. Oncology 1992; 49(suppl 2):28-31.

8 Perez MM, Newcomer AD, Moertel CG, Go VL, DiMagno EP. Assessment of weight loss, food intake, fat metabolism, malabsorption, and treatment of pancreatic insufficiency in pancreatic cancer. Cancer 1983;52:346-52.

9 DiMagno EP, Malagelada JR, Go VL. The relationships between pancreatic ductal obstruction and pancreatic secretion in man. Mayo Clin Proc 1979;54:157-62.

10 Abrams CK, Hamosh M, Dutta SK, Hubbard VS, Hamosh P. Role of nonpancreatic lipolytic activity in exocrine pancreatic insufficiency. Gastroenterology 1987;92:125-9.

11 Layer P, Peschel S, Schlesinger T, Goebell H. Human pancreatic secretion and intestinal motility: effects of ileal creatic secretion and intestinal motility: effects
nutrient perfusion. Am f Physiol 1990;258:G196.

12 Reuben DB, Mor V, Hiris J. Clinical symptoms and length Reuben $\mathrm{DB}$, Mor V, Hiris J. Clinical symptoms and length
of survival in patients with terminal cancer. Arch Intern Med of survival in patients

13 DeWys WD, on behalf of the Eastern Cooperative Oncology Group. Prognostic effect of weight loss prior to chemotherapy in cancer patients. Am $\mathcal{F}$ Med 1980;69:491-7. 